\title{
Adaptive Filtering for the Removal of Image Disturbances
}

\author{
Doron Benzvi \\ The Jerusalem College of Engineering \\ Jerusalem, Israel \\ doronb@jce.ac.il
}

\begin{abstract}
Adaptive filters are commonly used to remove disturbances corrupting a signal, particularly when a reference signal correlated to the disturbance is available. Accompanying random noise, sinusoidal disturbance or other undesired signals can be removed from the desired signal. When applied to images, the term adaptive filter commonly refers to a filter which changes its features based on the local statistical characteristics of the image and noise (within each image segment). It has also been proposed previously to use the LMS adaptive filter for 2-D image filtering, mostly for random noise removal. In this paper, it is offered to extend the use of the 2-D LMS adaptive filter to other image disturbances, such as a varying frequency sinusoidal disturbance, and the removal of an undesired added image from the desired one. Moreover, it is proposed here that in some cases it may be useful to convert the twodimensional image to a one-dimensional signal (by chaining its row or columns), and apply a 1-D LMS adaptive filter.

The 2-D LMS adaptive filter is demonstrated to work well in restoring an image corrupted by a random noise, a sinusoidal disturbance or a disturbance of an undesired image added to the desired one. In some particular conditions an adaptive filter with no reference signal may also be used to remove the undesired disturbance from the image.
\end{abstract}

Keywords: Adaptive Filtering, LMS algorithm, image restoration

\section{Introduction}

The adaptive filter for 1-D signals, see Figure 1, has two inputs: the signal with the added disturbance, $x(n)$, and a reference signal, $r(n)$, which is correlated with the disturbance $w(n)$ :

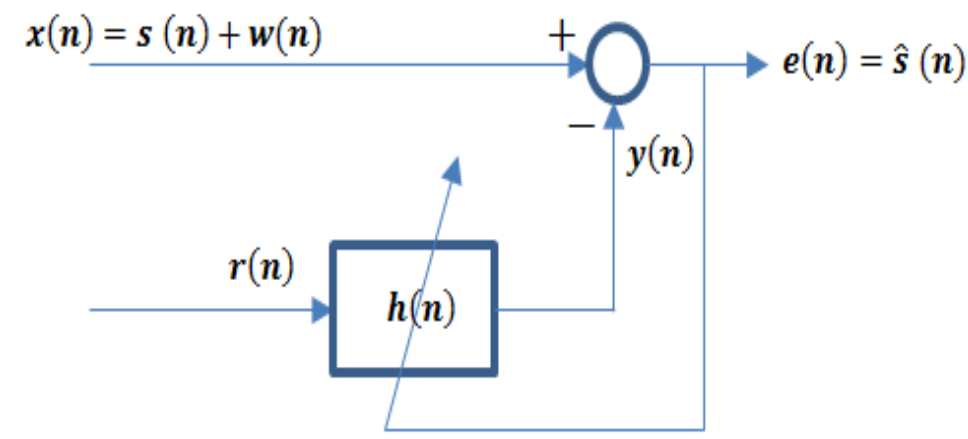

Figure 1. An Adaptive Filter for 1-D Signals

The filter coefficients are being updated based on the LMS algorithm [1]:

$$
h_{n}(k)=h_{n-1}(k)+\beta e(n) r(n-k), \quad k=0,1, \ldots, N-1
$$

where 
- $\quad N$ is the filter length (FIR)

- $\quad h_{n}(k)$ is the $\mathrm{n}$-th iteration of the $\mathrm{k}$-th filter sample

- $e(n)$ is the current output sample

- $\quad r(n-k)$ is a former reference sample

- $\quad \beta$ is the convergence coefficient

When the filter converges to its final version, the output, $e(n)$, is an estimate of the desired signal, $s(n)$, in the least square sense. Namely, the filter $h(n)$ obtained is the one producing minimal value to $\varepsilon=E\left\{e^{2}(n)\right\}$. At this state, the filter operating on the reference signal, $r(n)$, generates a signal close to the disturbance $w(n)$, and when subtracted from the signal with the disturbance, produces a close estimate to the desired signal $s(n)$.

The limitation of the adaptive filter is in the requirement to have a reference signal correlated with the disturbance. In certain cases, it is possible to have an adaptive filter with no additional reference signal, as in Figure 2:

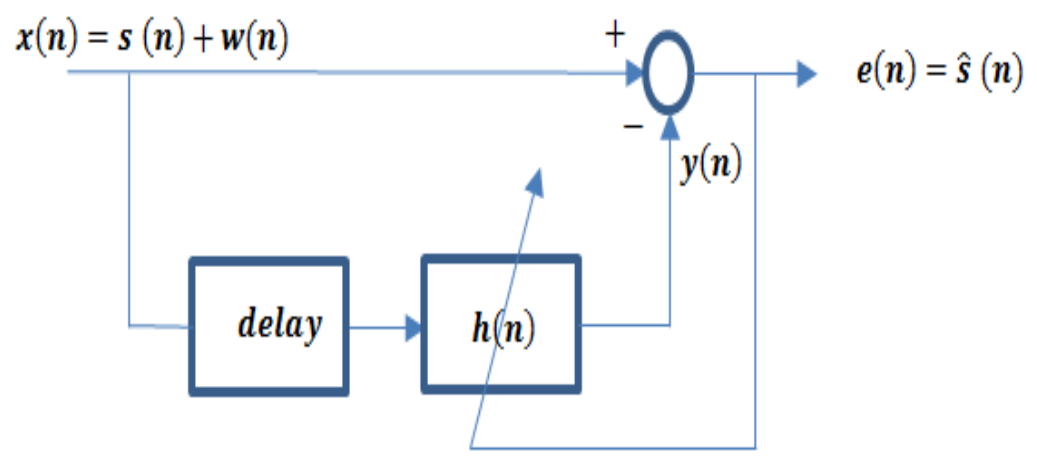

Figure 2. An Adaptive Filter with no External Reference Signal

This configuration is applicable when the desired signal $s(n)$ is a narrowband signal, and the disturbance $w(n)$ is a wideband signal, or vice versa. The delay added is designed to be greater than or equal to the correlation width of the wideband signal.

\section{Adaptive Filtering of 2-D Signals}

Two modes are offered to adjust the 1-D LMS adaptive filter to work with 2-D images: - chaining the image lines (or columns) into a 1-D signal, and applying the adaptive equation (1) above

- modifying eq. (1) above to work with a 2-D filter $h(m, n)$ and 2-D image inputs, a method suggested in [3].

\subsection{Converting the 2-D images into 1-D Signals}

The first mode of applying the 1-D adaptive filter to image data converts the 2-D image data to a 1-D signal by chaining the rows (columns) of the image, with the start of each image line following the end of the previous line. Once the image is converted to a 1-D signal, the common adaptive filter of eq. (1) is applicable. Then, the algorithm to update the adaptive filter coefficients proceeds in the same way as it does for 1-D signals, following eq. (1).

This mode is suitable, provided the adaptive FIR filter $h(n)$ is supposed to simulate a 1D filter be it horizontal or vertical. However, if the adaptive filter $h(n)$ is supposed to 
simulate the operation of a 2-D filter, then it will span a few (image) lines, and become too cumbersome to implement.

Consider the case where the disturbance is filtered by a filter $h_{r}(m, n)$ before being added to the desired image. Then, if the image with the filtered disturbance form one input to the adaptive filter, while the reference input is the original disturbance prior to being filtered, then, it is expected that the adaptive FIR filter $h(n)$ converges to $h_{r}(m, n)$. If $h_{r}(m, n)$ is of a size $N x N$, then $h(n)$ should be of a length larger then $N$ image lines. Hence, in these later cases, this mode of operation is not recommended.

\subsection{Modifying the Adaptive Filter Update Equation to Cope with 2-D Filters and Image Data}

The second mode of applying the 1-D adaptive filter to image data adjusts the known filter coefficient update eq. (1) to cope with 2-D filter and image data. As proposed in [2] the new form of the LMS equation is

$$
h_{i}(k, l)=h_{i-1}(k, l)+\beta e(m, n) r(m-k, n-l), \quad k=0, \ldots, N-1, l=0, \ldots, N-1
$$

where the filter $h(m, n)$ is of size $N_{x N}$, and the two inputs, the reference disturbance $r(m, n)$ and the image with the added disturbance $x(m, n)$, are both two-dimensional. $i$ is the iteration number, and for an $M x M$ image $i=m \cdot M+n$

The application of the LMS adaptive filter to 2-D image data has been used for noise removal [3]. Here, it is suggested to extend its use for other types of disturbances, such as sinusoidal disturbance (even with a varying frequency), and for cases where one undesired image is mixed with the desired one. Moreover, it is suggested that one-input adaptive filter (with no reference image), can also be useful for image restoration.

\section{Experimental Results}

The two modes of applying adaptive filtering to 2-D images have been tested on 3 types of disturbances:

- a random (white) noise

- a sinusoidal disturbance

- a disturbance of another image being superimposed on the desired one

\subsection{A Random White Noise Disturbance}

The adaptive filter is capable of removing the disturbance from the desired signal/image only if the reference signal/image is correlated with the added disturbance.

Thus, for a white noise disturbance, the adaptive filter is capable of restoring the desired image only if the reference image $r(m, n)$ and the added noise $w(m, n)$ are derived from the same noise sample - two white noise image samples are not correlated.

The results presented in this section are for the cases where

- the reference noise is a shifted and scaled version of the added noise

- the added noise $w(m, n)$ has been filtered by an unknown filter before being added to the desired image

In the first case the adaptive filter should converge to the shifting and scaling system. In the second case, the adaptive filter is expected to converge to the filter applied to the added noise.

Figure 3 shows the results of applying the adaptive filter when the reference random noise is a shifted version of the random noise added to the image. The shift in this case is both vertically and horizontally, and the 2-D LMS algorithm is applied. 


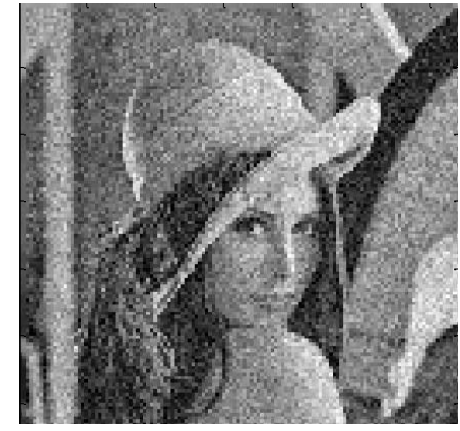

(a)

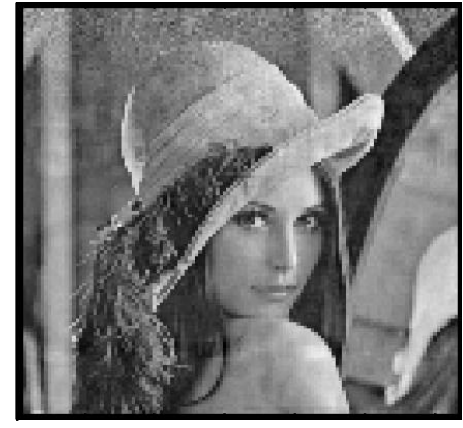

(b)

Figure 3. Restoring when the Reference Random Noise is a Shifted and Scaled Version of the Disturbance Added to the Image: (a) the Input Image with Additive Noise (b) the Restored Image

The shifting filter is applied to the added noise

$$
h=\left|\begin{array}{lllll}
0 & 0 & 0 & 0 & 0 \\
0 & 0 & 0 & 0 & 0
\end{array}\right|
$$

And the adaptive filter converges to

$$
h_{\text {adapt }}=\left(\begin{array}{lllll}
-0.0883 & -0.0289 & -0.0334 & -0.0193 & -0.0147 \\
-0.0266 & -0.0444 & -0.0286 & -0.0115 & -0.0034 \\
-0.0832 & -0.0864 & -0.0791 & -0.0762 & -0.0848 \\
-0.0413 & -0.0398 & -0.0378 & -0.0327 & -0.0374 \\
-0.0966 & -0.0928 & -0.0968 & -0.0710 & \mathbf{0 . 9 7 7 0}
\end{array}\right)
$$

The filter converges to the proper shifting filter, and the noise removed. Noise can still be seen at the top of the restored image in (b), before convergence to the proper filter.

Similarly, when the shift is only in one direction, the 1-D LMS algorithm is applied to the image after being converted to a 1-D vector by chaining its rows. The filter converges to the proper 1-D shifting filter, and the noise is largely removed from the image.

Figure 4 presents the results of applying the adaptive filter when the reference input is a random noise, and the noise added to the desired image is a filtered version of that random noise by a 2-D averaging filter of size $3 \times 3$. 


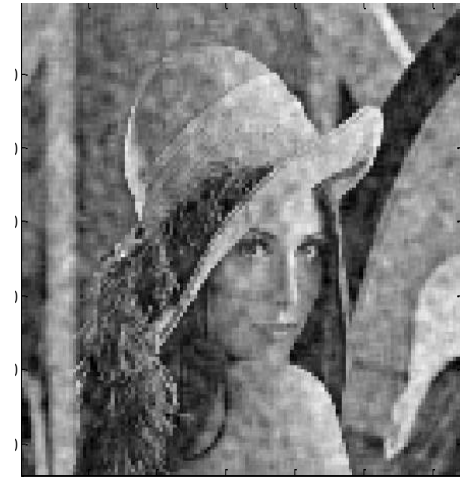

(a)

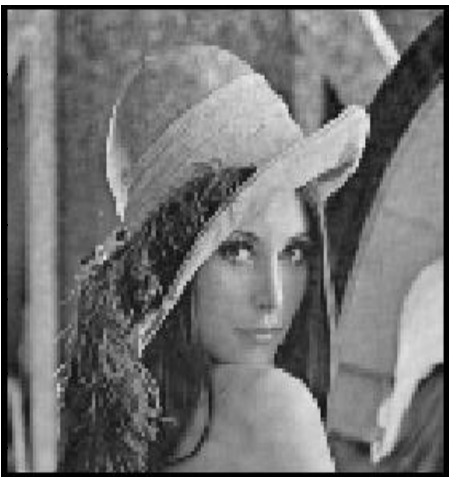

(b)

Figure 4. Restoring when the Random Noise Added to the Image is a Filtered Version of the Reference Noise: (a) the Input Image with Additive Filtered Noise (b) the Restored Image

The filter applied to the added noise is

$$
h=\frac{1}{9}\left(\begin{array}{lll}
1 & 1 & 1 \\
1 & 1 & 1 \\
1 & 1 & 1
\end{array}\right)
$$

And the adaptive filter converges to

$$
h_{\text {adapt }}=\left(\begin{array}{ccccc}
0.0130 & 0.0303 & 0.0221 & 0.0146 & 0.0213 \\
0.0054 & 0.1321 & 0.1266 & 0.1209 & 0.0240 \\
0.0091 & 0.1343 & 0.1307 & 0.1277 & 0.0264 \\
0.0081 & 0.1208 & 0.1260 & 0.1146 & 0.0088 \\
0.0039 & 0.0154 & 0.0224 & 0.0102 & 0.0133
\end{array}\right)
$$

The filter converges to the proper $3 \times 3$ averaging filter, and the noise is removed. Noise can still be seen at the top of the restored image in (b), before converging to the proper filter.

In a similar manner, when the averaging is only in one direction, the 1-D LMS algorithm is applied to the image after being converted to a 1-D vector by chaining its rows. The filter converges to the proper 1-D averaging filter, and the noise is largely removed from the image.

\subsection{A Sinusoidal Disturbance}

For a sinusoidal disturbance, the adaptive filter is capable of restoring the desired image only if the reference image $r(m, n)$ and the added noise $w(m, n)$ are of the same spatial frequency. Thus, the results presented in this section are for cases where the reference sinusoidal disturbance $r(m, n)$ is a shifted and scaled version of the added disturbance $w(m, n)$, namely, they differ in their amplitude and phase.

Figure 5 presents the results of applying the adaptive filter when the reference input is a sinusoidal disturbance, and the disturbance added to the desired image is a shifted and scaled version of that disturbance. 


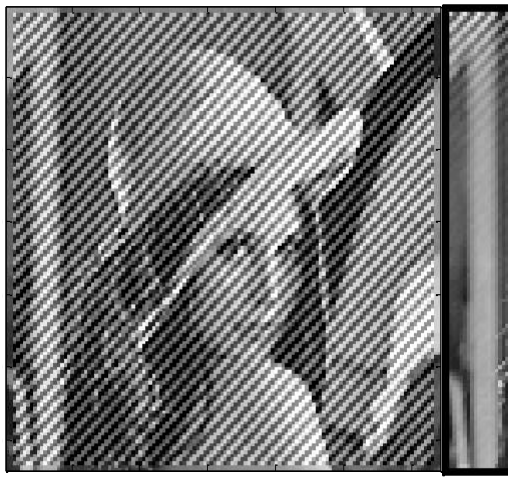

(a)

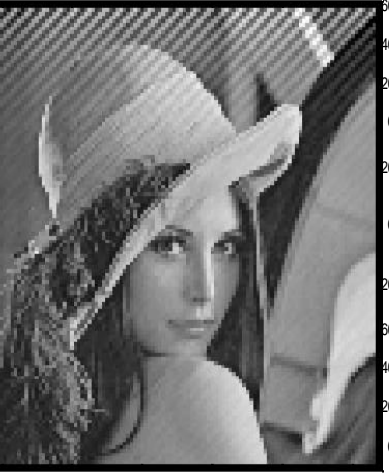

(b)

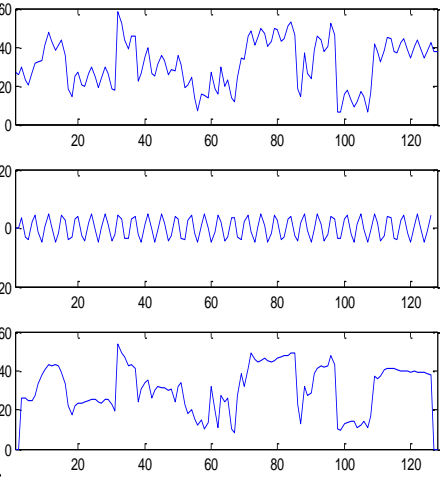

(c)

Figure 5. Restoring when the Sinusoidal Disturbance Added to the Image is a Shifted and Scaled Version of the Reference Sinusoidal Input (a) The Image with the Disturbance (b) the Restored Image (c) One Line from Input Image (Top), the Reference Sinusoidal Image (Middle) and the Restored Image (Bottom) with the Disturbance Removed

The filter converges to the proper shifting and scaling filter, and the disturbance removed. Sinusoidal noise can still be seen at the top of the restored image - before converging to the proper filter. The filter is also useful, when the frequency of the disturbance varies within the image as in Figure 6. There, the spatial frequency of the disturbance at the upper half of the image is twice that at the bottom half (in both directions). The varying disturbance is properly removed. Sinusoidal noise can still be seen at the top and middle of the restored image, before the filter converges to its final state.

Similarly, adequate results are obtained when the added disturbance is a filtered version of the reference input, and the adaptive filter converges to the correct applied filter.

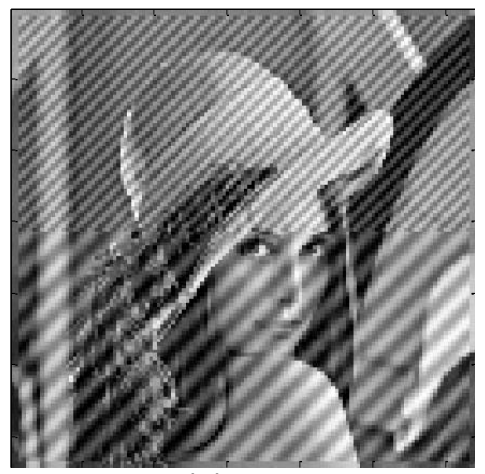

(a)

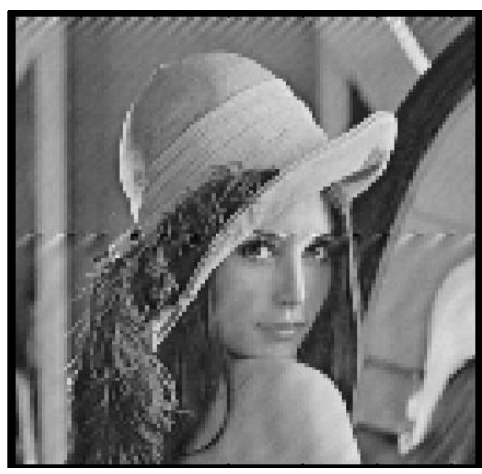

(b)

Figure 6. Restoring when the Sinusoidal Disturbance Added to the Image is of a Varying Frequency: (a) The Image with the Disturbance (b) The Restored Image

\subsection{A Superimposed Image Disturbance}

Adaptive filtering is also applicable in cases where the undesired disturbance is another image superimposed on the desired image. The undesired image can be removed provided a reference image correlated to it is available. The following results refer to the case where the reference image is a shifted and scaled version of the disturbance image added 
to the desired one. The same configuration is also useful when the added disturbance is a filtered version of the reference image.

Figure 7 presents the results when the image disturbance, the musical notes, is first shifted and scaled before being added to the desired image of fishermen. The reference image disturbance is the musical notes image. The filter converges to the proper shifting and scaling filter, separating the undesired image from the desired one. Image disturbance can still be seen at the top of the restored image - before the convergence of the filter.

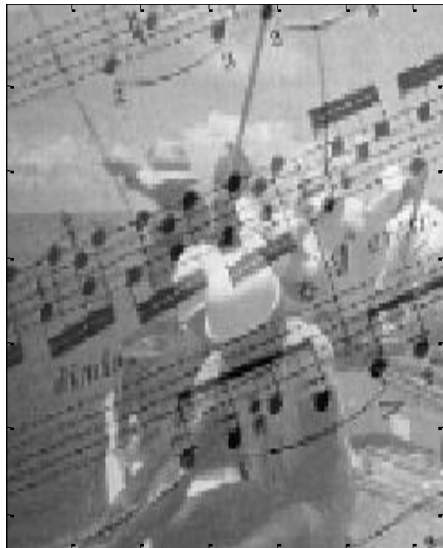

(a)

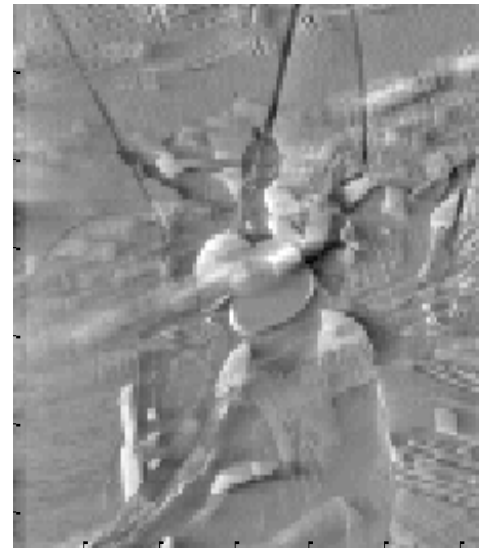

(b)

Figure 7. Separating Images using Adaptive Filtering (a) the Combined Image (b) the Restored Desired Image

\subsection{Results for Applying a Single-input Adaptive Filter to Corrupted 2-D Images (No Reference Input)}

An adaptive filter with no reference image is applicable when the image and the disturbance differ in their bandwidth, i.e. one is wideband and the other is narrowband. The delay in Figure 2 is of the correlation width of the wideband component of the input. When the disturbance is a white noise (the wideband component), a sufficient value for the delay is 1 , regardless of the direction (be it horizontal, vertical or both).

Figure 8 presents the results of applying a single-input adaptive filter when a white noise disturbance is added to the desired image, as in (a). The adaptive filter converges to a low-pass filter, producing the image in (b), with an improved signal to noise ratio. The image in (c), with the high frequencies, is generated at the overall output, after subtracting the image in (b) from that of (a). Lines from the original image (top), improved image (middle) and the difference image (bottom) are displayed in (d).

The same single-input adaptive filter structure is used to remove a narrowband sinusoidal disturbance from the relatively wideband image. In this case, the wideband desired image has a wider correlation width. Hence, the delay used (for the filter structure of Figure 2) is larger than 10. The restored image is obtained in this case at the overall output from the filter, while at the adaptive filter output the sinusoidal disturbance is isolated. 


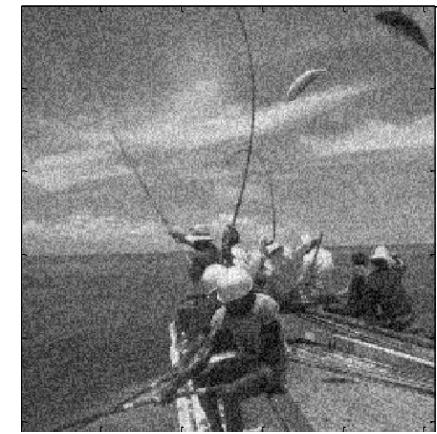

(a)

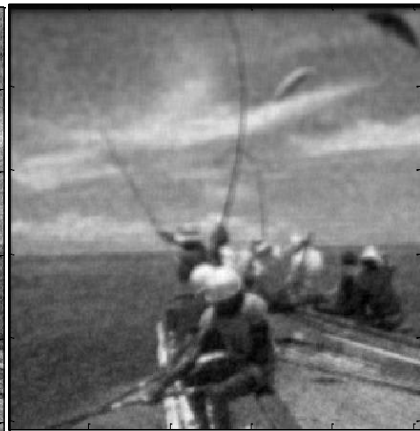

(b)

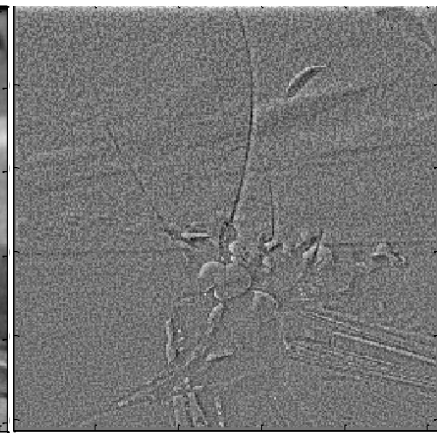

(c)
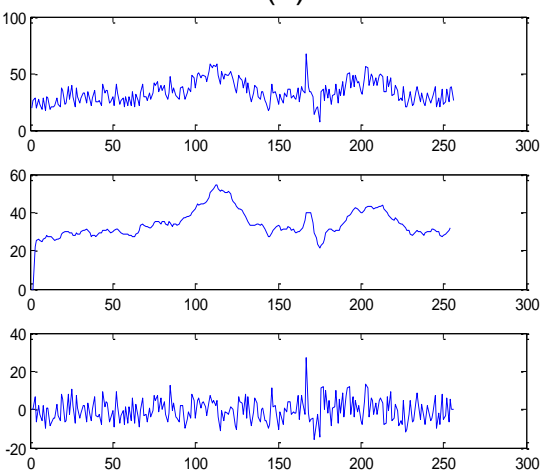

(d)

Figure 8. The Results of Applying a Single-input Adaptive Filter when a White Noise Disturbance is Added to the Desired Image, as in (a) (b) The Improved Image (c) the Difference Image (d) a Single Line from the 3 Images

Figure 9 presents the results of applying a single-input adaptive filter when a sinusoidal disturbance is added to the desired image, as in (a). The adaptive filter converges to a band-pass filter, centered at the sinusoidal disturbance frequency, as in the bottom graph of (c). The restored image is that of (b).

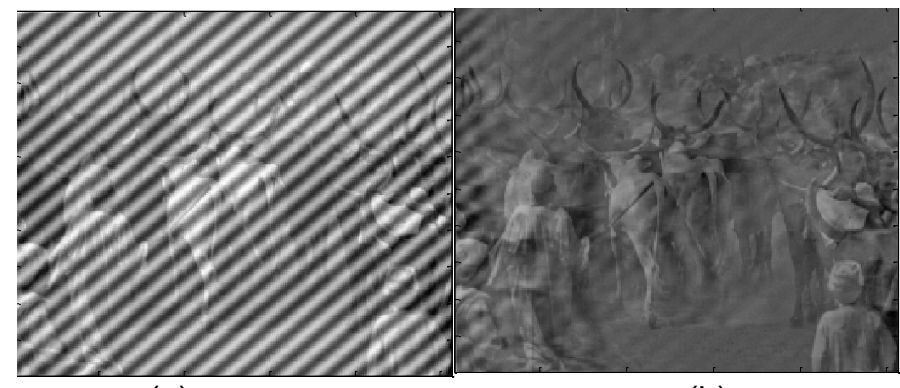

(a) (b)

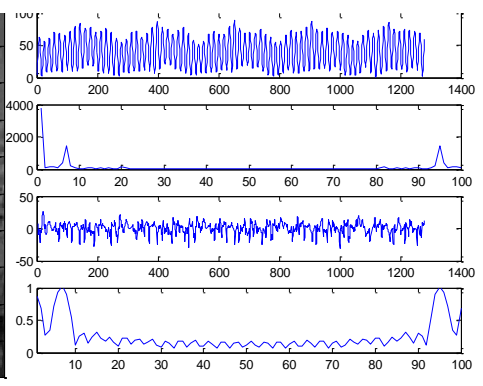

(c)

Figure 9. The Results of Applying a Single-input Adaptive Filter when a Sinusoidal Disturbance is Added to the Desired Image, as in (a). (b) The Improved Image at the Overall Output (c) a Single Line from the Original Image (Top) and its Transform (Second from Top), a Single Line from the Restored Image (Second from Bottom), and the Final Filter Converging to a Band-pass (Bottom)

\section{Conclusion}

The two-input adaptive filter, commonly used for signal restoration, is applied in this paper to 2-D image restoration. It has been demonstrated that given a reference image, 
correlated with the added disturbance image, the desired image can be restored. Random noise, a sinusoidal disturbance and an undesired superimposed image can be removed by the 2-D LMS adaptive filter, converging to the correct value. In the particular cases, where the image and disturbance differ in their spectral width, a single input adaptive filter with no reference image can be applied. Moreover, in cases where the reference image has undergone a 1-D deformation before being used, such as a shift in one direction or filtering by a 1-D filter, the 1-D LMS adaptive filter can be used for 2-D images by converting them to $1-\mathrm{D}$ vectors.

\section{References}

[1] S. O. Haykin, "Adaptive Filter Theory", $4^{\text {th }}$ Edition, Prentice Hall, New Jersey, (2002).

[2] P. Chan and J. S. Lim, "One-dimensional processing for adaptive image restoration", IEEE transactions on Acoustics, Speech and Signal Processing, vol. 33, no. 1, (1985), pp. 117-129.

[3] M. M. Hadhoud and D. W. Thomas, "The two-dimensional adaptive LMS filter algorithm", IEEE transactions on Circuits and Systems, vol. 35, no. 5, (1988), pp. 485-494. 
International Journal of Signal Processing, Image Processing and Pattern Recognition Vol. 8, No. 5 (2015) 